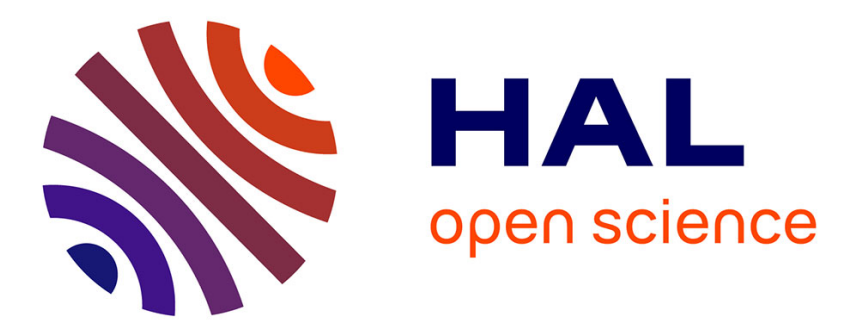

\title{
On the use of (U)RANS and LES approaches for turbulent incompressible single phase flows in nuclear engineering applications
}

Sofiane Benhamadouche

\section{- To cite this version:}

Sofiane Benhamadouche. On the use of (U)RANS and LES approaches for turbulent incompressible single phase flows in nuclear engineering applications. Nuclear Engineering and Design, 2017, 312, pp.2-11. 10.1016/j.nucengdes.2016.11.002 . hal-01715567

\author{
HAL Id: hal-01715567 \\ https://hal.science/hal-01715567
}

Submitted on 22 Feb 2018

HAL is a multi-disciplinary open access archive for the deposit and dissemination of scientific research documents, whether they are published or not. The documents may come from teaching and research institutions in France or abroad, or from public or private research centers.
L'archive ouverte pluridisciplinaire HAL, est destinée au dépôt et à la diffusion de documents scientifiques de niveau recherche, publiés ou non, émanant des établissements d'enseignement et de recherche français ou étrangers, des laboratoires publics ou privés. 


\title{
On the use of (U)RANS and LES approaches for turbulent incompressible single phase flows in nuclear engineering applications
}

\author{
S. Benhamadouche ${ }^{\mathrm{a}, *}$ \\ ${ }^{a}$ Electricité de France, R\&D Division, 6, Quai Watier, 78401 Chatou, France \\ * Corresponding author. Email address: sofiane.benhamadouche@edf.fr
}

\begin{abstract}
The present paper gives some ideas and guidelines one should keep in mind while running (U)RANS or LES computations. The paper starts with (U)RANS approaches, advocating the use of Reynolds Stress Models for complex flows and recommending further work on modeling of turbulent heat fluxes, which remains today too basic in industry. The superiority of wallresolved models vs. wall-modeled in RANS is recalled and the use of adaptive wall treatment is suggested. The concept of Unsteady RANS is finally questioned.

Then, important issues around LES are raised. The mesh refinement criteria are recalled for wall-resolved LES and the use of wall models addressed. The production of DNS and wallresolved LES calculations for flow understanding and RANS validation is encouraged.
\end{abstract}

\section{Keywords}

LES, (U)RANS, Nuclear engineering, Wall-resolved, Wall-modeled, HPC

\section{Introduction}

(Unsteady) Reynolds Averaged Navier-Stokes ((U)RANS) and Large Eddy Simulation (LES) approaches are today widely used in nuclear engineering applications for predicting the dynamics and heat transfer of turbulent flows. Both approaches exist since a long time and will remain useful for understanding several industrial configurations for several decades, playing different roles. The present overview is restricted, for the sake of clarity, to (U)RANS and LES for configurations in which the flow is incompressible and single phase as we consider that a lot of work is still required for this type of flow and for these two approaches.

This article is in the continuity of an invited lecture by Chabard and Laurence (2009) at Turbulence Heat and Mass Transfer conference 6. In this contribution, the authors insisted on the importance of RANS approaches in general and in particular on Reynolds Stress Models (RSMs) without forgetting the role of LES. Detached Eddy Simulation (DES), Scale-Adaptive Simulation (SAS) ... turbulence modeling approaches are out the scope of the present article. Verification and Validation $(\mathrm{V} \& \mathrm{~V})$, which is a necessary procedure to check the correct numerical solution of the equations and the ability of a model to predict standard flows is a prerequisite but will not be addressed. Neither can Uncertainty Quantification (UQ) but the hundreds of simulations required for the latter make fine LES unaffordable, incompatible today, with UQ. The statements given in the following largely assume the use a standard finite volume approach as this is the most common discretization used in nuclear engineering. Regarding heat transfer, nothing particular will be said for very low Prandtl numbers and one assumes values around 1 which is the case in Pressure Water Reactors (PWR). Finally, as turbulence modeling is full of acronyms, the author does his best to reduce them and to recall them, at least for the first use.

On the one hand, steady and unsteady RANS turbulence models are frequently used in nuclear engineering applications as they are more affordable than finer approaches. While looking at 
the literature, it is obvious that the users are more inclined to use Linear Eddy Viscosity Models (LEVMs), especially stable and widely known/recommended ones such as the Realizable k- $\varepsilon$, the k- $\omega$ SST ... This is legitimate, but are they the best models to be used in nuclear engineering configurations? Why our use of RSMs is still so marginal? Wall-resolved models, often called Low Reynolds Number models (LRN) exist for both Eddy Viscosity Models (EVMs) and RSMs. These models are still rarely used due to their cost. Is it possible today to avoid the use of wall functions/boundary layer modeling, at least in some applications? While performing heat transfer computations, as several computations are done using LEVMs, almost all the contributions use a Simple Gradient Diffusion Hypothesis (SGDH) which utilizes the concept of the turbulent Prandtl number. Limitations of such assumptions will be shown later. Is the concept of wall functions/modeling really avoidable, in particular for large computational domains or very high Reynolds numbers? One can find several contributions using an Unsteady RANS when LES is difficult to carry out or unaffordable and when quantifying the unsteadiness is needed such as in Fluid Structure Interaction or thermal fatigue issues. Is the spectral content of a URANS computation always meaningful?

On the other hand, LES is becoming more and more utilized thanks to the growth of High Performance Computing (HPC) resources (speed, memory, storage). The present article focuses on standard use of LES using sub-grid scale eddy viscosity concept as it is the one widely used in industry. What are the space discretization criteria that make us confident in a LES simulation? Due to standard limitations in computational resources, wall-modeled LES using wall functions or even hybrid RANS/LES approaches are still the solution to tackle high Reynolds numbers. Is this approach realistic? Finally, few words are said about HPC projections in the near future as this is one of the major evolutions that one may expect, in particular for LES.

\section{2. (U)RANS approaches}

\subsection{Regarding the use of LEVMs vs. RSMs}

Linear Eddy Viscosity Models might be very useful, even combined with wall functions, in order to obtain reliable global quantities such as pressure drops, mass flow rates, averaged Nusselt numbers ... Many examples exist in the literature showing that these approaches are sufficient to reasonably predict these kinds of quantities, or even more local ones, such as mean velocity or mean temperature profiles. Wells et al. (2015) give an overview of a benchmark organized by EPRI where several models have been used to predict the flow through a fuel assembly. LEVMs seem sufficient to predict the pressure drop through a grid with mixing vanes (see Conner et al., 2015 who show that the Realizable k- $\varepsilon$ gives a good estimate of the pressure drop through a mixing vane grid). However, Benhamadouche (2015) shows that the utilized RSM (SSG model, see Speziale et al., 1991), on the same configuration, is very sensitive to the discretization scheme and produces good results with an accurate numerical scheme. Thus, the RSMs are much more sensitive to the discretization errors than the EVMs and should be used with special care. One can find in the literature conclusions such as "The Reynolds Stress Model gives poor results compared to the k- $\varepsilon$ "! As the latter is just the trace of the first one, this is not intellectually speaking satisfactory. Something must be wrong with the use of the model (numerical approach, inlet boundary conditions ...). Furthermore, many of those using RSMs in the literature rarely give details about the specific model used. One may find sentences such as "The RSM has been tested ...". On the contrary, when they use an EVM, the precise version of the model is given; standard k- $\varepsilon$ (Launder and Spalding, 1974), Realizable k- $\varepsilon$ (Shih et al., 1995), k- $\omega$ SST (Menter 1994) ... There are several RSMs in the literature; among them the LRR (Launder et al., 1975) and the SSG (Speziale et al., 1991), with several variants that may affect the constants or some terms in the Reynolds stresses and dissipation equations (see Pope 
2000). There are also numerous test cases and publications and even a whole book (Hanjalić and B. Launder, 2011) demonstrating the necessity of resorting to RSMs when body forces (buoyancy, rotation, swirl) induce specific anisotropy to the stress which is then no longer aligned on the mean velocity gradient. These remarks show that, on the one hand, RSMs (or the use of RSMs) are probably not mature enough in an industrial context and, on the other hand, they need to be used more often in order to obtain a wealth of feedback similar to the one existing for EVMs. Bellet and Benhamadouche (2010) focused on the importance of turbulence modeling to predict swirling flows. The case shown in Fig. 1 clearly emphasizes the inability of EVMs, even advanced ones such as the $\mathrm{v}^{2} \mathrm{f}$ (here the $\varphi=\mathrm{v}^{2} / \mathrm{f}$ - model introduced by Laurence et al., 2004), to predict the circumferential flow in a vortex tube. The test-case is taken from Escudier et al. (1980) (see Fig. 1 top). The swirl is globally created in the large cylinder then goes inside the small one. Even at high small to large tube diameter ratios, EVMs are unable to correctly predict the flow structure (see Fig. 1 bottom). However, the Reynolds Stress Model (SSG model here with Scalable Wall Functions, see Esch and Menter, 2003) and the LES predict the flow rather well. Note that for small diameter ratios, only LES is able to predict the vortex breakdown phenomenon which appears. The ability of LES to predict such flows has been already shown by Derksen (2005) and is confirmed here. This kind of example shows that when rotating or secondary flows play a major role, such as the flow through mixing grids or in the hot legs of a PWR upper plenum, a second moment closure (RSM) should be used by default in order to capture the anisotropy of turbulence and, hence, obtain satisfactory local quantities. A last argument used sometimes to justify the use of an EVM instead of a RSM is the cost of this latter. As for incompressible solvers, the pressure correction step in much more expensive than solving transport equations, this is in the author's opinion an argument out of date.

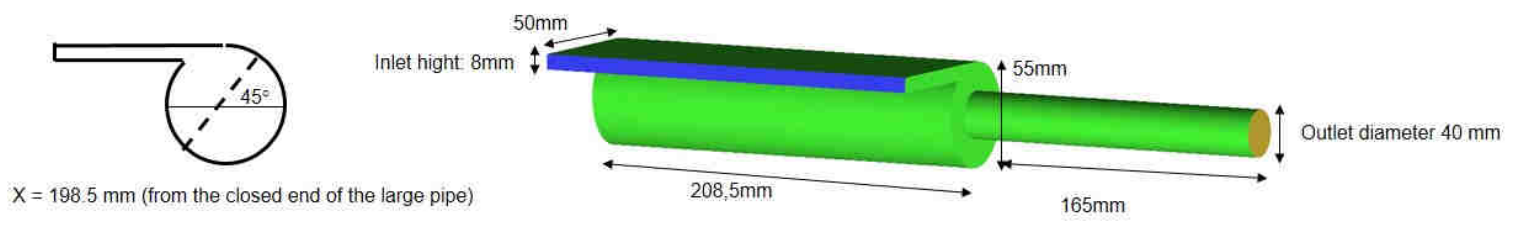

left: dashed line along which the profiles are plotted, right: test-case dimensions)
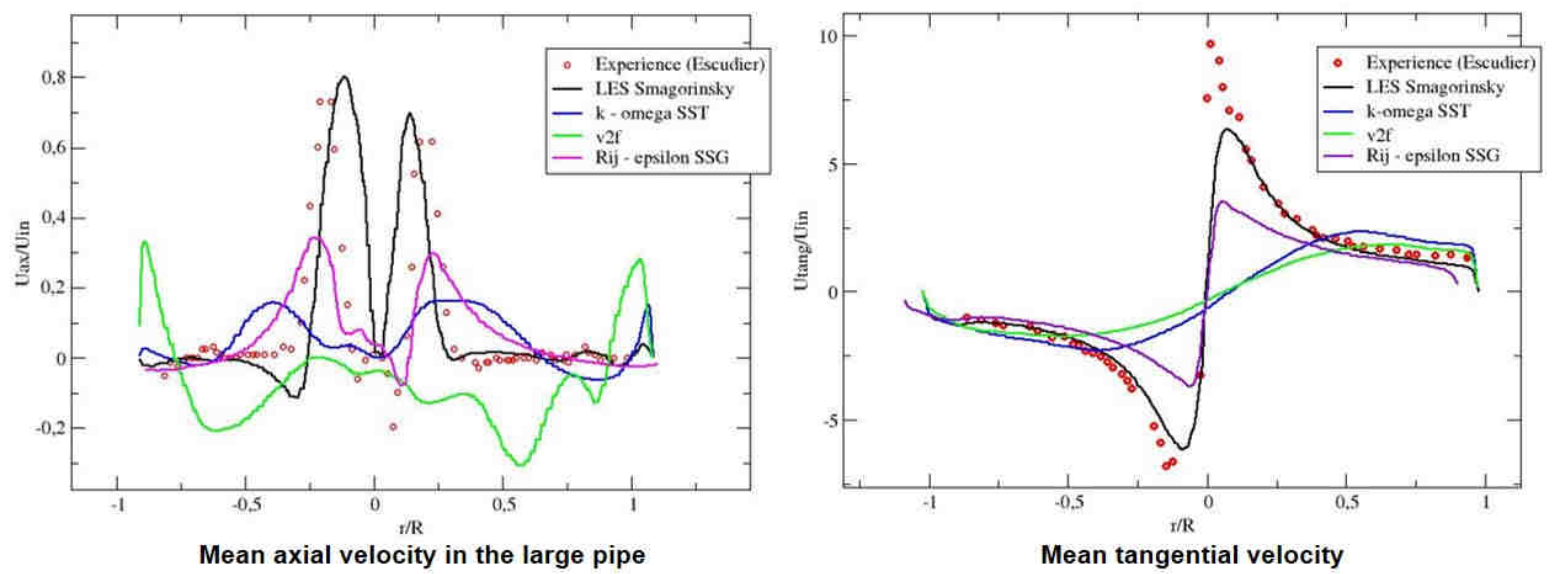

Fig. 1. Top: confined Vortex Tube Geometry, Re(inlet) $=1900$, ratio of angular to axial momentum $\Omega=4.14$, bottom: mean axial and tangential velocity in the large pipe $(198.5 \mathrm{~mm}$ from the closed end). 


\subsection{Wall-resolved vs. wall-modeled RANS approaches}

Utilizing a wall-resolved RANS model (often called in specialized literature Low Reynolds Number (LRN) models) is becoming more and more affordable when the Reynolds number is moderate to high (depending on the definition of the Reynolds number, this gives Reynolds numbers going from $10^{4}$ to $10^{7}$ ). This kind of models is obviously better than the one using a wall-modeled approach at the wall. If one uses the same wall-resolved RANS model (such as the k- $\omega$ SST from Menter (1994), the k- $\varepsilon$ from Launder and Sharma (1974), the $v^{2} f$ from Durbin (1991) or one of its more robust versions, the Elliptic Blending Reynolds Stress Model from Manceau and Hanjalić (2002)), as a basis, one can't expect obtaining better results with a wall model either with an algebraic approach (so called "wall functions" approach, see Craft et al., 2002) or even with a numerical one (solving 1D differential equations in the near wall cell, see Craft et al., 2004). Thus, when it is possible, the calculation should be done with a wall-resolved model, fulfilling the well-known requirement $y+<1$ everywhere. Here, $y+$ is the nondimensional distance to the wall of the first computational cell based on the friction velocity and the kinematic viscosity. Of course, a grid refinement study has to be carried out in order to obtain a numerically converged result.

However, even if the case can be, theoretically speaking, computed using a wall-resolved RANS model, the complexity or the size of the geometry may lead, depending on the meshing strategy, to regions where it is impossible to refine and thus which need a wall treatment. Solutions to mix wall-resolved and wall-modeled-regions, called "adaptive wall treatment" in a single mesh have been developed by Popovac and K. Hanjalić (2007) and Billard et al. (2015) for EVMs and by Wald et al. (2015) for RSMs. A mesh cut downstream a mixing grid in a fuel assembly is shown in Fig. 2. The meshing strategy uses here a block-structured method with ICEM CFD. One can see the traces of the dimples and springs and the variations in the refinement obtained around a rod. This shows the importance of developing adaptive wall treatments, either with wall functions or a 1D numerical approaches, departing from a wallresolved model.

Wall-modeled approaches might give satisfactory results in some configurations, in particular for predicting global quantities. This is not always true, that is why a grid sensitivity study is highly recommended whenever one uses this kind of approaches. An example is given in Fig. 3. Several computations (not all shown here but the reader can refer to Benhamadouche et al., 2015a, 2015b) have been performed for the flow through a square edged orifice plate at a Reynolds number equal to 25000 , based on the bulk velocity $U_{b}$ in the main pipe and the diameter of this latter. The ratio of the orifice diameter to the pipe diameter is $\beta=0.62$, and the ratio of the orifice thickness to the pipe diameter is 0.11 . The target value is the discharge coefficient through the contraction. Fig. 3 shows the discharge coefficient measured $1 D$ upstream of the orifice and $0.5 D$ downstream (both measured from the upstream face of the contraction as required in ISO 5167-2, 2013). EVMs, not shown here, exhibit similar mesh sensitivity as the wall-modeled RSM utilized here (the SSG of Speziale et al., 1991 combined to the standard two velocity scales wall function). The ISO correlation estimate obtained from ISO 5167-2 (2013) represented in the figure gives the target value (however, only the behavior of the numerical results is important here). The wall-resolved RANS approach uses the Elliptic Blending (EB) concept introduced by Manceau and Hanjalić (2002) with SSG Reynolds Stress Model far from the walls (note that the same concept exists for EVMs, see Billard and D. Laurence, 2012). This model is inspired from elliptic relaxation introduced by Durbin (1993) but using only one elliptic equation to model the wall damping instead of six, one for each 
Reynolds stress as initially suggested. This wall-resolved RANS model is called EB-RSM for Elliptic Blending-Reynolds Stress Model. Fig. 3 shows the discharge coefficient across the orifice as a function of the refinement. Note here that the characteristic non dimensional mesh size in the abscissa is representative of the wall normal one. When the mesh is refined in the wall normal direction, it is also refined in the stream-wise and azimuthal directions using the same ratios. Two different meshes are used for the wall-modeled approach (see Benhamadouche et al., 2015b) For the coarsest refinement of Mesh2, the non-dimensional wall distance $y+$ is approximately 55 upstream of the orifice and increases up to $y+\approx 150$ where the flow reattaches. For the finest refinement of Mesh2, y+ $\approx 22$ upstream of the orifice and $y+\approx 60$ where the flow reattaches. The non-dimensional wall distances for the refinements of Mesh1 lie within the range of those of Mesh2. The corresponding values for the coarsest mesh of the wall-resolved model are $y+\approx 1$ upstream of the orifice and $y+\approx 2$ where the flow reattaches. The refinement ratio between two successive meshes is 1.3 and is applied equally in the streamwise, radial and azimuthal directions. As one can notice, the wall-resolved model results are almost grid independent, whereas wall-modeled approach based on the same homogeneous model (SSG model far from the walls), exhibits a strong sensitivity to the mesh refinement. In the present case. Only the wall-modeled approach is able to predict a discharge coefficient not very sensitive to the mesh refinement and within less that $1 \%$ of the ISO correlation value (a wall-resolved LES in Benhamadouche et al. (2015a) gives a discharge coefficient very close to the one given by the ISO).

This example shows the systematic and logical superiority of wall-resolved or Low Reynolds Numbers approaches compared to wall modeled ones (algebraic or numerical wall treatment). However, wall-resolved approaches will probably remain impossible for decades to use systematically either for very large computational domains (a whole nuclear vessel for example) or for very large Reynolds numbers (such as in the hot legs of a $1300 \mathrm{MWe}$ where the Reynolds number is of the order of $10^{8}$ ). In these cases, the use of specific near wall treatments, such as the "adaptive wall treatment" suggested above, will remain mandatory for many years.

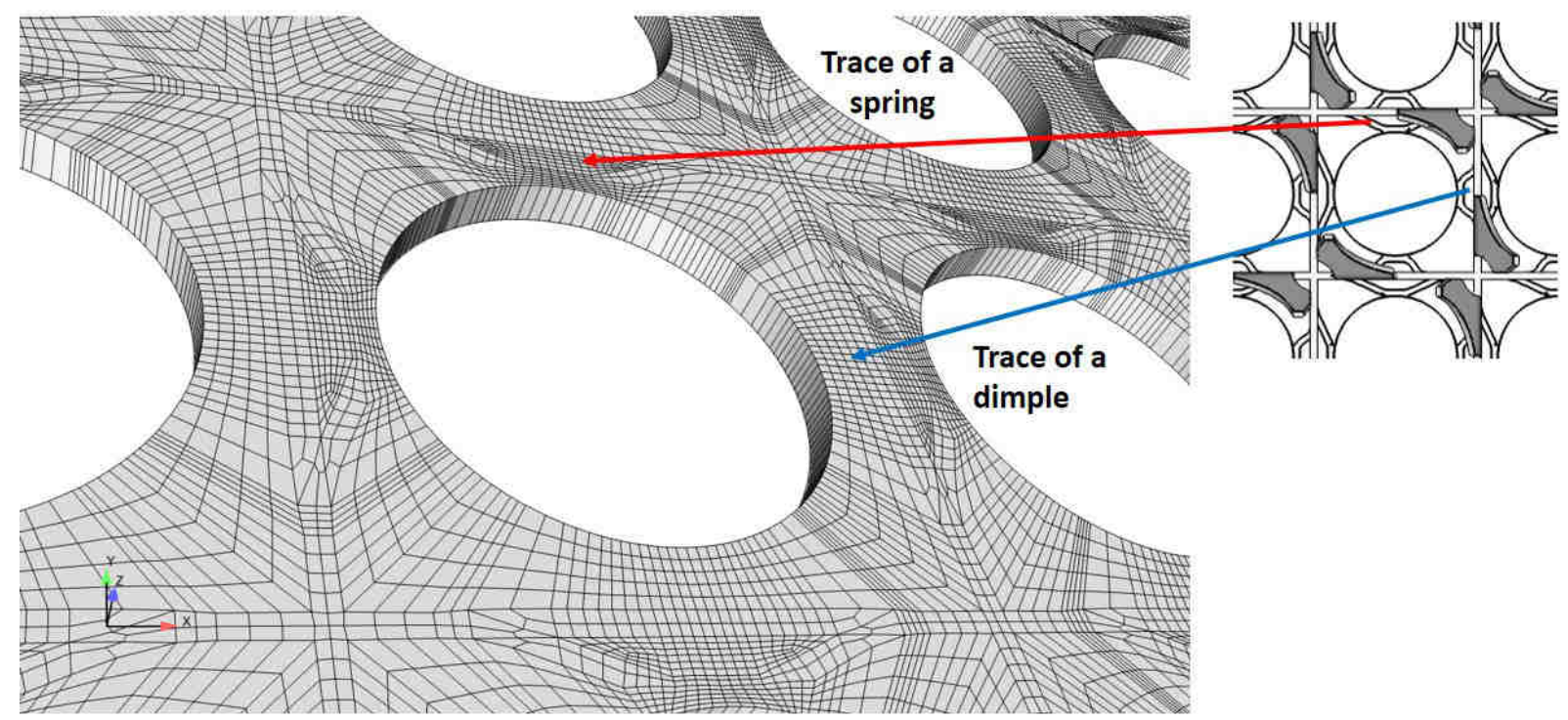

Fig. 2. Mesh cut downstream a grid with mixing vanes. 

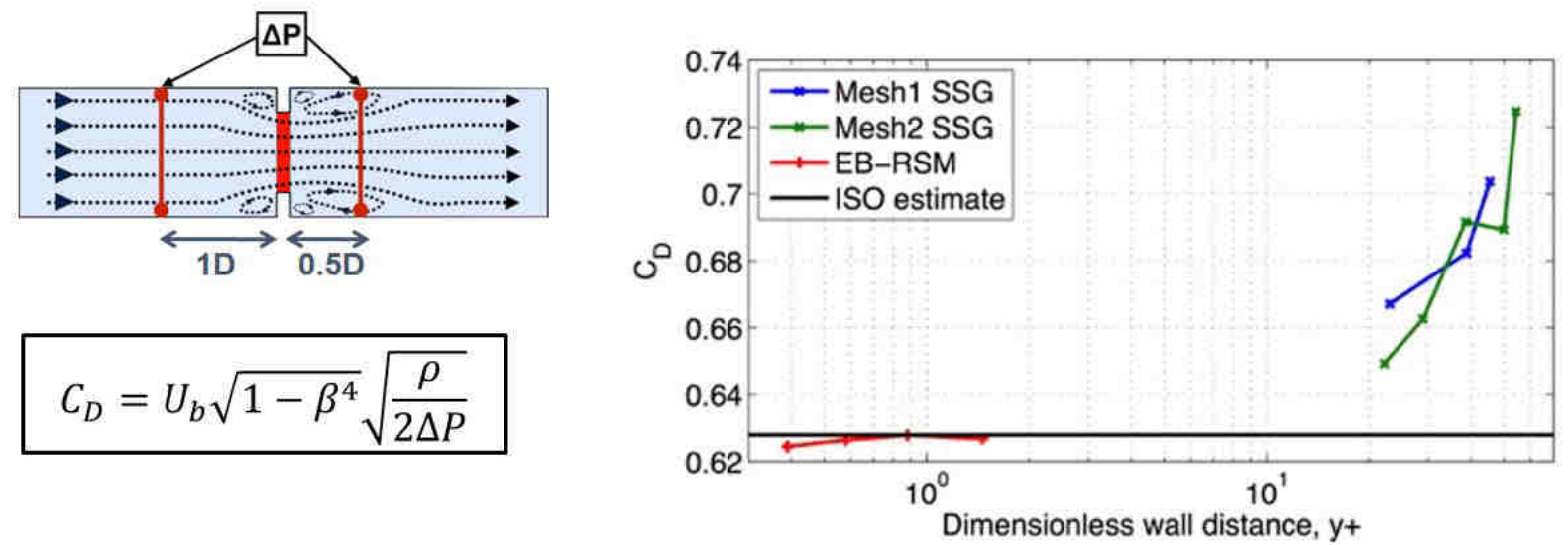

Fig. 3. Left: Computational domain for the square edged circular orifice plate, right: discharge coefficient across the orifice versus mesh refinement.

\subsection{Heat transfer predictions with RANS approaches}

\subsubsection{With Eddy Viscosity Models}

As a majority of industrial contributions still use EVMs and particularly, LEVMs, one starts by these models. It is impressive to see the number of users who still utilize the Simple Gradient Diffusion Hypothesis (SGDH) approach even with buoyancy effects. It is possible with an EVM to increase models' capabilities by introducing more sophisticated models (see Kenjereš et al., 2005) such as the Generalized Gradient Diffusion Hypothesis (GGDH), the AFM (Algebraic Flux Model) which can take into account buoyancy effects, or even more advanced models based on transport equations for the turbulent heat fluxes. The little interest for modeling the turbulent heat fluxes has several reasons. First, the lack of experimental or Direct Numerical Simulation data compared to the ones available for the dynamics. The lack of experimental data can be explained by the difficulties one may face to carry out such experiments and the inherent financial cost. Some valuable DNS data have been made available for turbulent flows with heat transfer (see Kasagi and Nishimura, 1997 and Trias et al., 2007) among others) but much more efforts are still needed in particular for conjugate heat transfer cases. For the latter, DNS data is rare, with the exception of the work done by Tiselj et al. (2013) and the ongoing work by Flageul et al. (2015), whereas with current DNS codes and resources, the scientific community could easily be more prolific in this area.

The next example is often studied as coolant flows in the cores of current gas-cooled nuclear reactors which contain ascending vertical flows in a large number of parallel passages (see Keshmiri et al., 2012). The flow going upwards in a vertical heated pipe is the analogous academic test case. The flow properties are considered constant and the Boussinesq approximation is used. While increasing buoyancy, the flow experiences a re-laminarization. This transition, which may occur in other situations and which dramatically affects heat transfer, may be found in other industrial configurations. Both Kim et al. (2008) and Keshmiri et al. (2012) studied this flow with EVMs and Fig. 4 gives a fair illustration of the results. They concluded that several EVMs fail in predicting the mixed convection regime. One may notice in particular that the widely used k- $\omega$ SST exhibits good results for low and high buoyancy parameter values but totally fails in predicting the transitional regime. Note that the computations have been carried out with two different codes (see Fig. 4). More recent models such as the ones based on elliptic relaxation concepts tend to better predict such flows as they include wall normal velocity mean fluctuations and turbulent length-scale characteristics 
$\left(\varphi=\mathrm{v}^{2} / \mathrm{f}\right.$ model results in Keshmiri et al., 2012). Introducing more physics in the EVMs seems then necessary to better predict heat transfer phenomena.

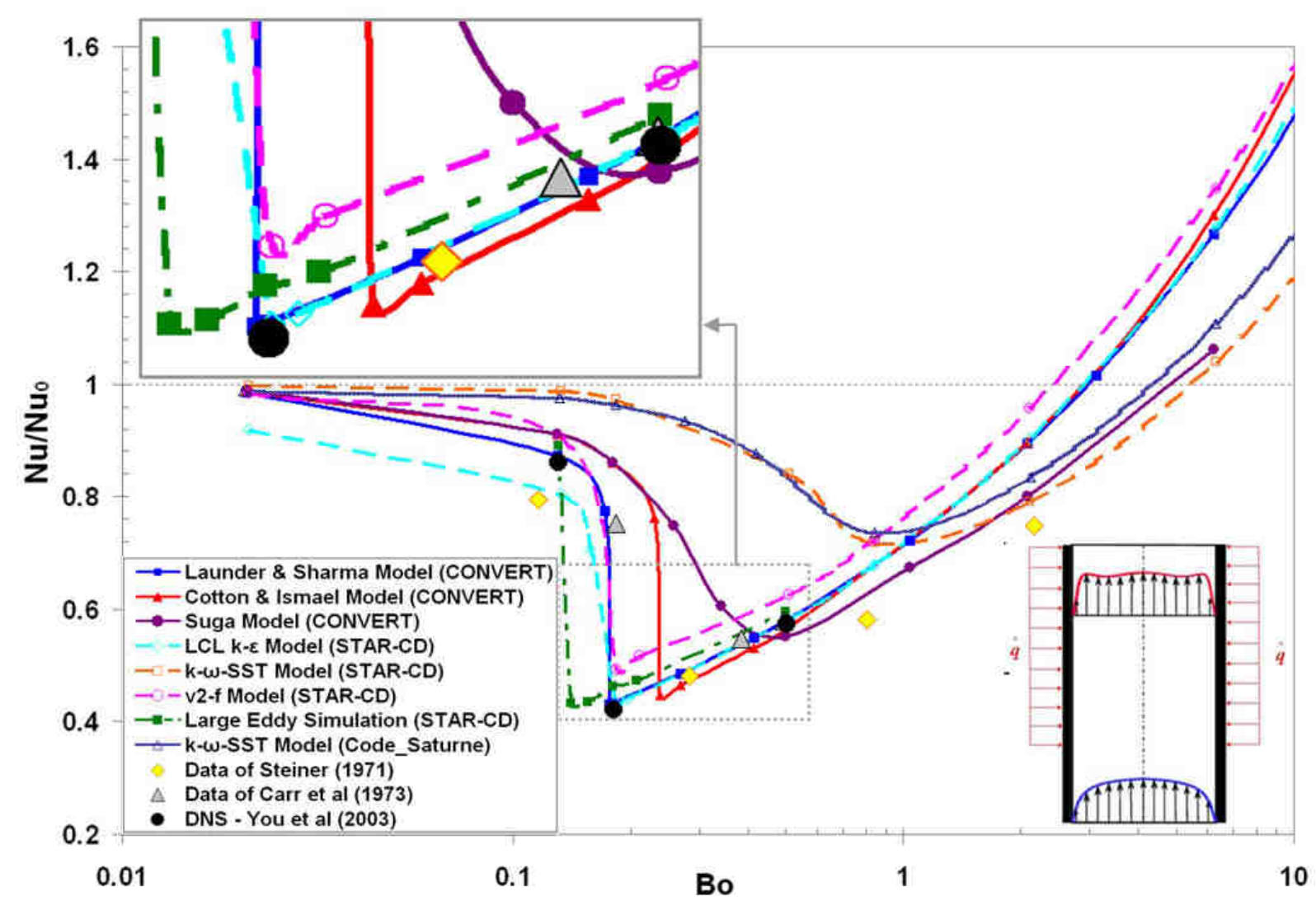

Fig. 4. Ratio of mixed-to-forced convection Nusselt number as a function of the buoyancy parameter by several RANS models, LES and DNS for an ascending mixed convection flow (from Keshmiri et al., 2012).

\subsubsection{Going beyond Eddy Viscosity Models}

Another way to enrich the physics is to use a RSM combined with an advanced treatment for the turbulent heat fluxes using at least a GGDH or an AFM or if possible a Differential Flux Model (DFM, see Dol et al. (1999) among others) in which transport equations are solved for the turbulent fluxes. Several authors already introduced these approaches but these are still far from mainstream. Recently, Dehoux et al. (2011) and Dehoux (2012) used the GGDH, AFM and DFM in conjunction with the EB-RSM (Elliptic Blending-Reynolds Stress Model) and extended the elliptic blending concept for modeling the turbulent heat fluxes. Fig. 5 shows the numerical results obtained with different approaches for the temperature variance in a turbulent channel flow in the forced and mixed convection regimes. These profiles show a progressive enhancement of the temperature variance as the turbulent heat flux model is enriched (from SGDH to DFM, then, from EB-GGDH to EB-DFM, see Dehoux (2012)). Note that the model here used for the dynamics is the EB-RSM. One may also notice the poor behavior of the two advanced EVMs ( $\mathrm{k} \omega \mathrm{SST}$ and $\varphi$-model) in the mixed convection regime.

Other more complex flows, not shown here, such as the one in a heated cavity and a PWR upper plenum and its hot legs confirm the need of advanced models for the turbulent heat fluxes, at least algebraic ones. 

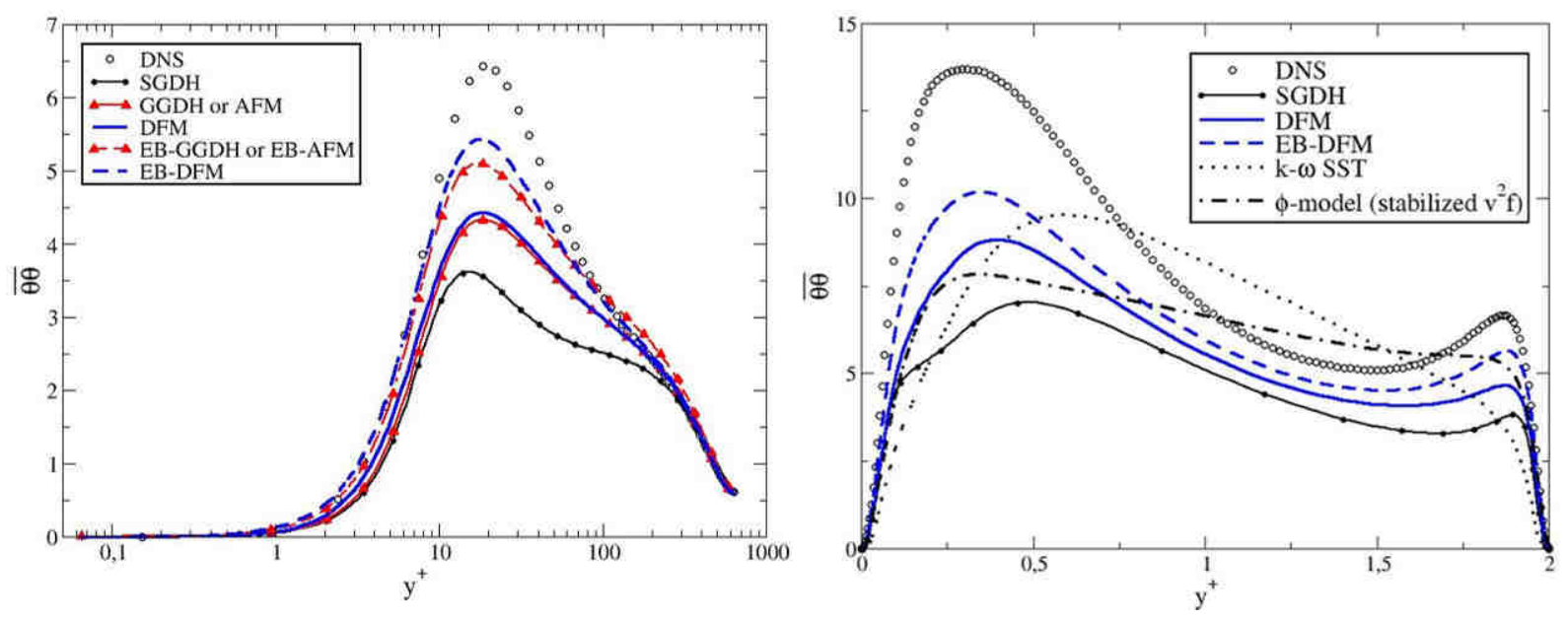

Fig. 5. Temperature variance. Left: forced convection, channel flow, $\operatorname{Re}_{\tau}=640, \operatorname{Pr}=0.71$, comparisons to DNS data from Abe et al. (2004). Right: mixed convection, channel flow, $\operatorname{Re}_{\tau}=150, \mathrm{Gr}=9.610^{5}, \operatorname{Pr}=0.71$, comparisons to DNS data from Kasagi and Nishimura (1997).

\subsection{On Unsteady RANS}

RANS equations in their unsteady form (so called URANS) are widely used when unsteadiness is needed. Their use for example in fuel bundle configurations is widespread in particular while aiming at predicting gap instabilities (see Chang and Tavoularis, 2008 and Meyer 2010). The approach is attractive since DNS or even LES are still impossible to carry out with standard computational resources at the core Reynolds numbers (see Tavoularis, 2011). First, as it has been reported by Tavoularis (2011), one should be very cautious about the interpretation of the regular coherent structures predicted in URANS. The question raised hereafter is about the meaning of unsteadiness with URANS. The following example shows that if one observes unsteadiness with URANS on a given mesh, it might disappear while refining the mesh and in this case what started out as URANS ends up as a steady state RANS. Of course, the instability of the following case has nothing to do with gap instability but shows that a popular case which has been considered for a long time as a URANS case, in particular with RSMs, is actually a RANS one and no unsteadiness should be expected. Fadai-Ghotbi et al. (2008) computed the 2D backward facing step flow and proved that the unsteadiness of the vortex shedding decreases while refining the mesh by using a wall-resolved RSM. They also showed that 3D computations give a steady converged solution. Fig. 6 shows the maximum value of the ratio resolved energy/total energy as a function of the stream-wise location while progressively refining the mesh (from mesh 7 to 11 , mesh 12 giving a steady solution). This example clearly campaigns for verifying the evolution of the resolved energy while performing mesh sensitivity/convergence studies. 


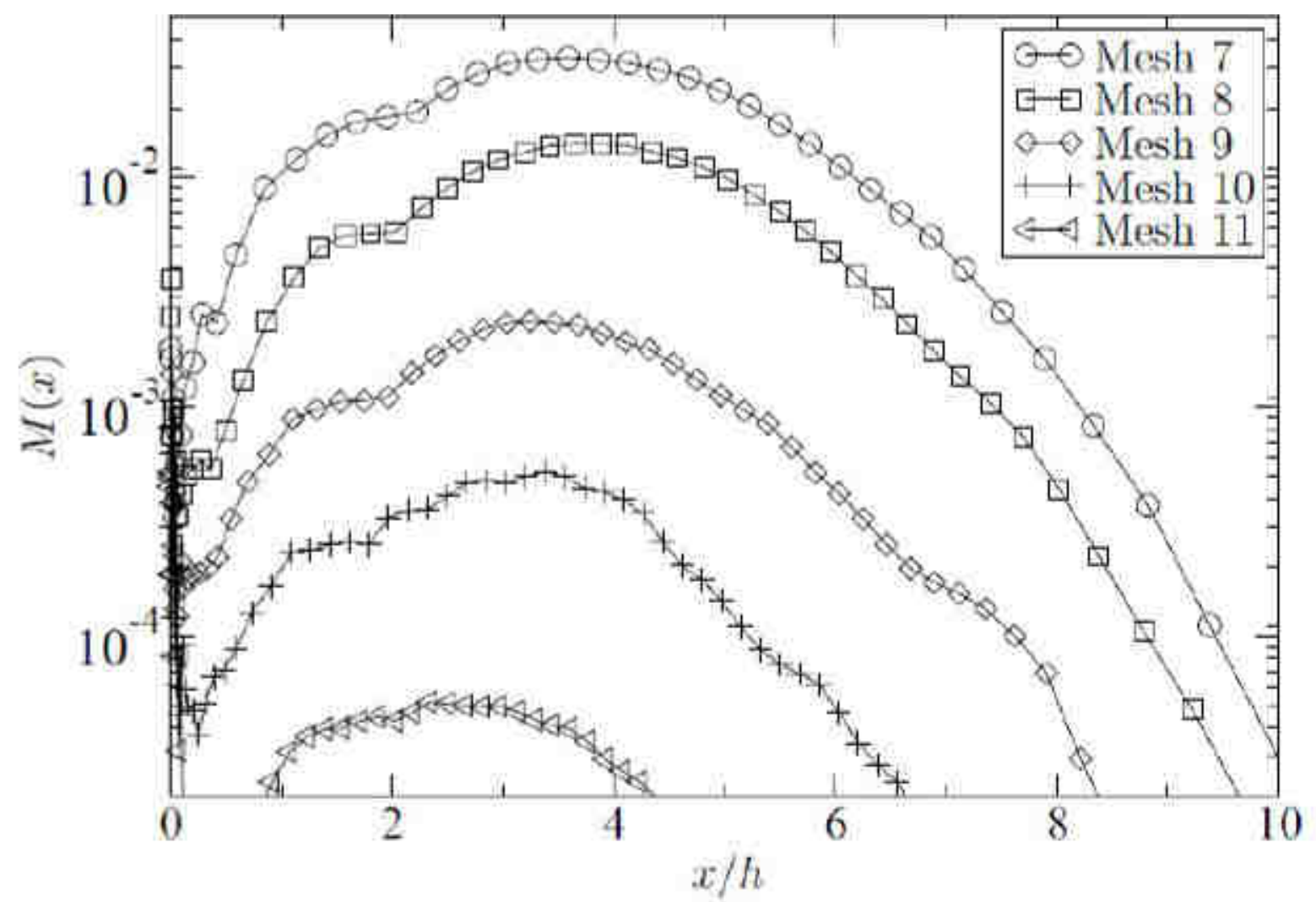

Fig. 6. Maximum resolved energy/total energy ratio as a function of the stream-wise location for successive mesh refinements. Mesh 12, finest, gives a steady solution. Image taken from Fadai-Ghotbi et al. (2008).

\section{LES approaches}

\subsection{Quasi-DNS and wall-resolved LES}

More and more wall-resolved LES are found in the literature (see for example Afgan et al., 2011). These computations are sometimes called Quasi-DNS (Komen et al., 2014) or even DNS (Baglietto et al., 2006) providing valuable data, such as Reynolds Stresses budgets or turbulent heat fluxes (see Shams et al., 2013), for RANS modeling. However, in the author's opinion, the nomenclature is not adequate as in numerous examples the discretization is only second order in space or less as sometimes a bounded central differencing scheme is introduced for the convection term. Rhie and Chow interpolation in the pressure correction step is utilized with the collocated arrangement and introduces additional numerical errors. These computations should rather be called wall-resolved LES or very fine LES. With the growth of computing power and thanks to advanced numerical techniques, proper DNS computations for complex flows, higher Reynolds numbers and even more complex geometries are today available (see for example Ranjan et al., 2011 and Dairay et al., 2015, among others) and there is a hope that this will continue in the future.

\subsection{Wall resolved LES space refinement requirements}

Still several communications dealing with wall-resolved LES insist on the refinement in the wall normal direction $(\Delta y+<1)$ but not on the stream-wise and span-wise ones. It is commonly admitted today that for a turbulent channel flow and using a second order discretization scheme, one has to respect the two additional criteria; $\Delta \mathrm{x}+<40$ and $\Delta \mathrm{z}+<20$, where $\mathrm{x}$ and $\mathrm{z}$ are the streamwise and span-wise coordinates, respectively. Fig. 7 gives an illustration of the results one may obtain while only refining in the wall normal direction without taking care of the other directions (here $\Delta \mathrm{x}+$ and $\Delta \mathrm{z}+$ go from 100 to 1000). With this computation, the predicted shear 
stress is too important due to artificial streaks that appear at the wall. The computation using wall functions (with the same refinement in $\mathrm{x}$ and $\mathrm{z}$ directions) but a first computational point that satisfies $30<\Delta y+<100$ gives better results.

However, the wall refinement is not the only criteria that should prevail. One has ideally to check whether the computational cells are all in the inertial sub-range, let's say of the order of the Taylor-micro length scale. One may perform a precursor RANS computation which gives of course reasonable results. This computation can give a reasonable estimate of the Taylor micro-scale and the LES mesh can then be created (one should take care of locations where the flow exhibits too low turbulent Reynolds numbers). The example of an estimate of the Taylor micro-scale is given in Fig. 8 for the flow along a ribbed wall. The LES mesh has been created based on this scale and the computation gave very satisfactory results (see Keshmiri et al., 2016).

A posteriori checks should also be performed in order to check whether the turbulent kinetic energy is reasonably resolved. Afgan et al. (2011) showed this kind of a posteriori estimations by computing an estimate of the sub-grid scale dissipation rate and turbulent kinetic energy (see Fig. 9). This estimate is much better and has more sense than using the ratio of the sub-grid scale viscosity to the kinematic viscosity. If the estimated sub-grid scale turbulent kinetic energy is everywhere lower than 5\% of the total turbulent kinetic energy, one can consider that the Large Eddy Simulation is fine enough.

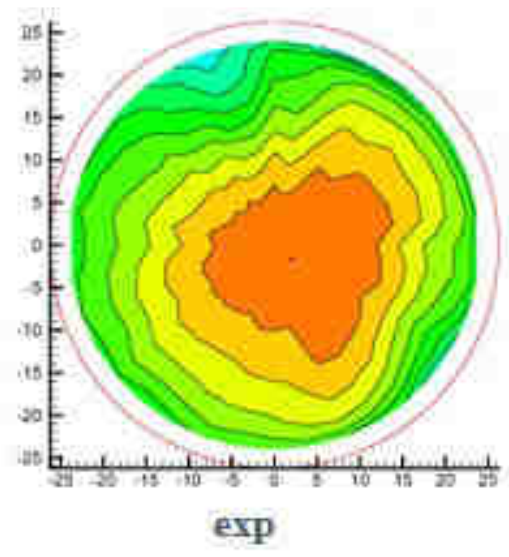

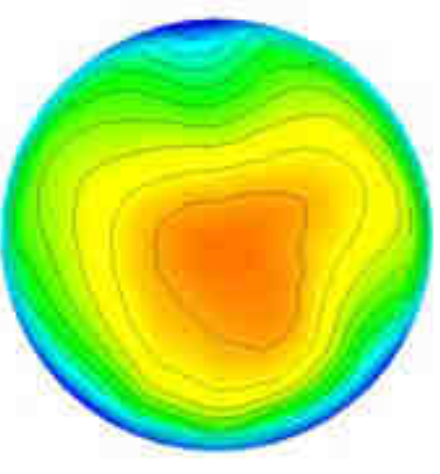

Wall modeled

LES

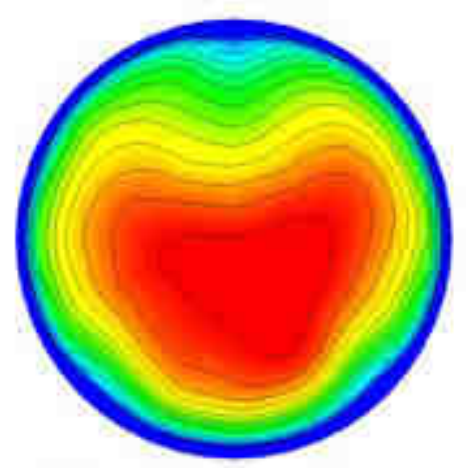

LES with $\Delta \mathrm{y}^{+} \sim 1$

$\Delta \mathrm{x}^{+}, \Delta \mathrm{z}^{+} \sim 100$ to 1000

Fig. 7. Mean stream-wise velocity component downstream a T-junction. 

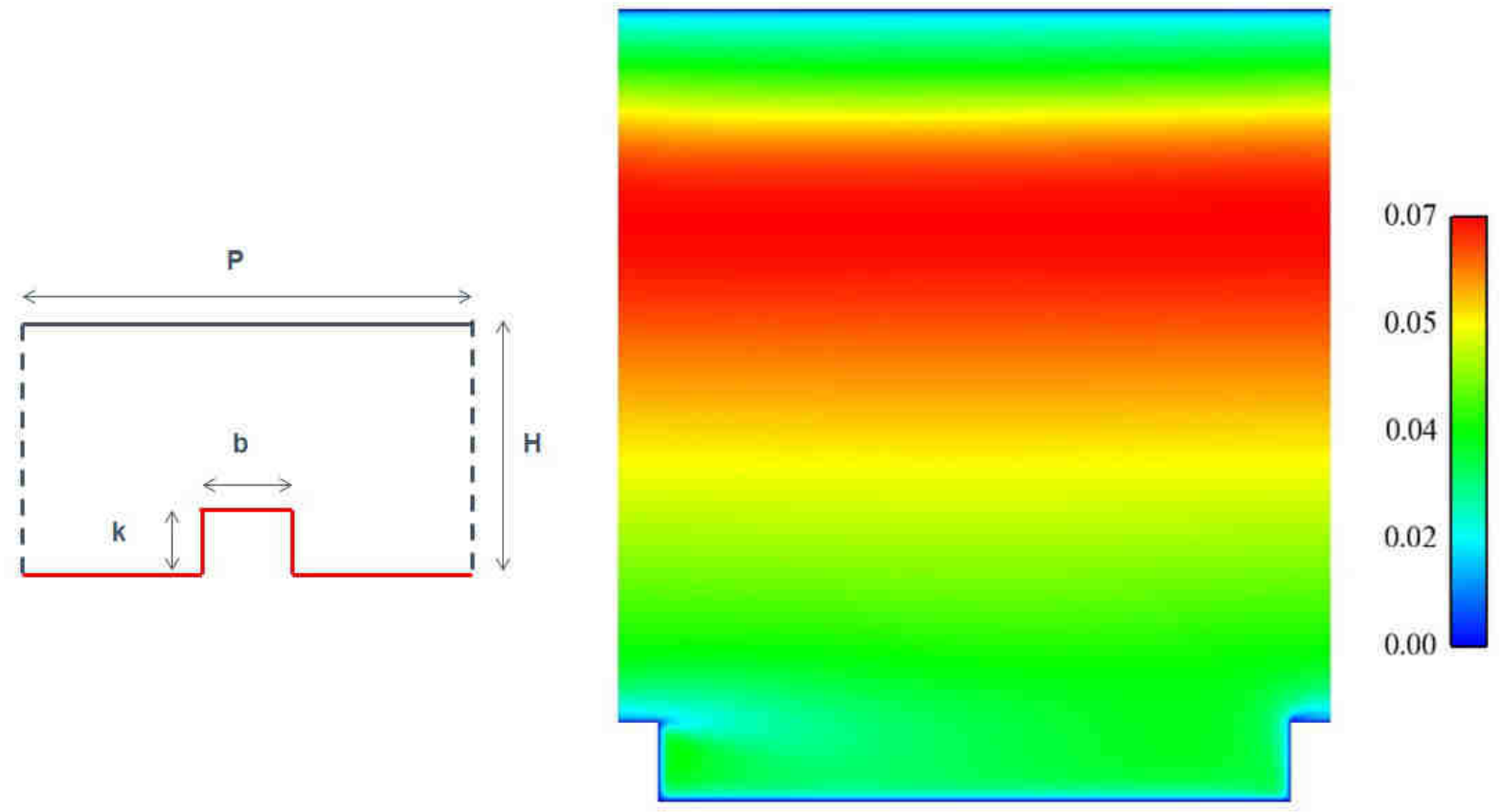

Fig. 8. Flow along a ribbed wall. $R e=30000$ with $P / k=9, H / k=10, b / k=1$. The Taylor microscale predicted by the $\varphi$-model (Laurence et al., 2004).
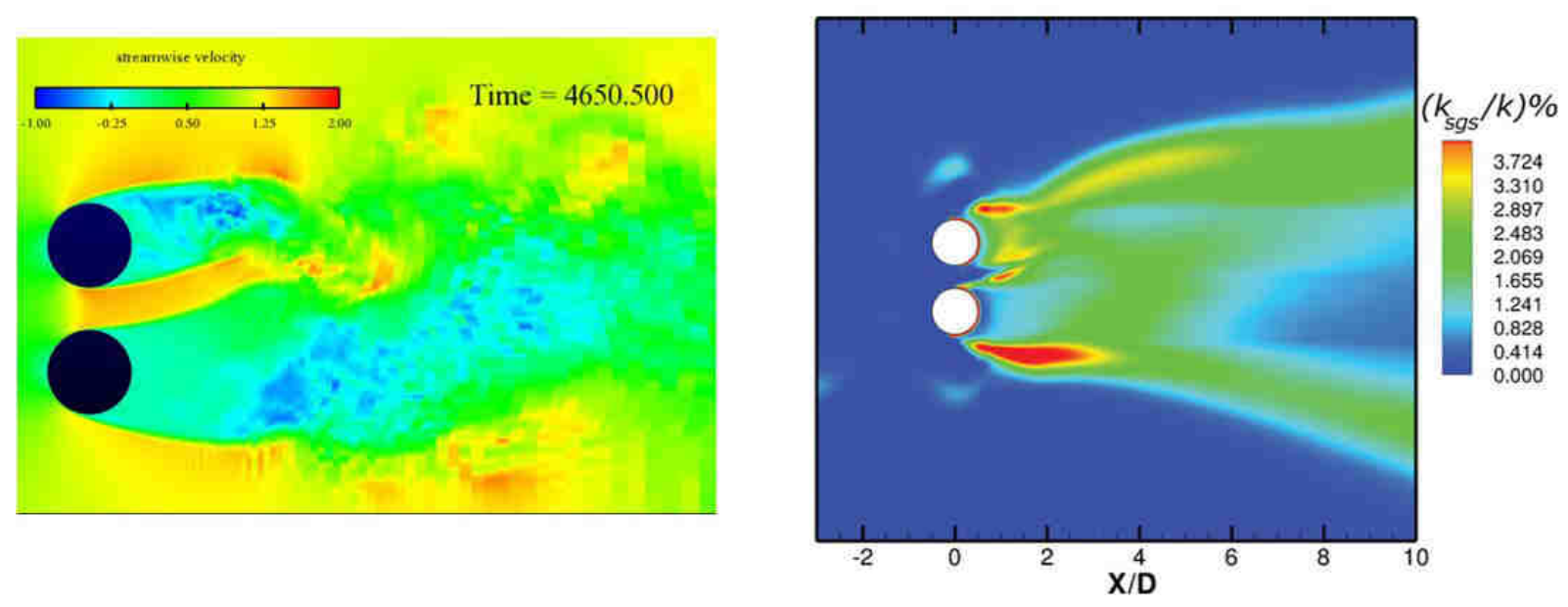

Fig. 9. The flow around two side-by-side cylinders at subcritical Reynolds numbers $(\mathrm{Re}=3000)$. Estimates of the sub-grid scale turbulent kinetic energy $\left(k_{S G S}=\frac{3}{2} c_{K}\left(\frac{\varepsilon_{S G S} \Delta}{\pi}\right)^{2 / 3}\right.$ with $\varepsilon_{S G S}=\overline{\left.v_{t} \frac{\partial \tilde{u}_{i}^{\prime}}{\partial x_{j}} \frac{\partial \tilde{u}_{i}^{\prime}}{\partial x_{j}}\right)}$

\subsection{Wall modeling in LES?}

As it has been shown in Fig. 7, wall-modeled LES might give correct mean values. However, its use is still subject to discussion and objections (see Piomelli, 2008). This approach, which 
often uses wall functions concepts, is a very first step of hybrid RANS/LES technique (using a logarithmic profile for example). Considering the complexity of the flow dynamics in the near wall region, there is rather weak correlation between an instantaneous velocity at a given distance from the wall and the instantaneous shear-stress under it and only long time averaged values are well rendered, by construction. This approach is thus a priori not recommended for unsteady problems such as thermal fatigue or Fluid Structure Interaction. However, in order to deal with realistic Reynolds numbers, one can test this approach and check whether the low frequencies predicted at the first computational cell are sufficient for the studied problem. Fig. 10 gives an example taken from Benhamadouche et al. (2009) in which the objective is to compute the pressure load along a rod bundle in a typical fuel assembly at $\operatorname{Re}=40000$, based on the hydraulic diameter and the bulk velocity. The pressure load has been used combined to a beam equation to predict the displacements obtained along the central rod and the qualitative results correspond to what is observed in reality (few microns). However, this computation can't be considered as validated and the methodology has to be confronted to experimental data (or why not DNS data with the available computing power today).
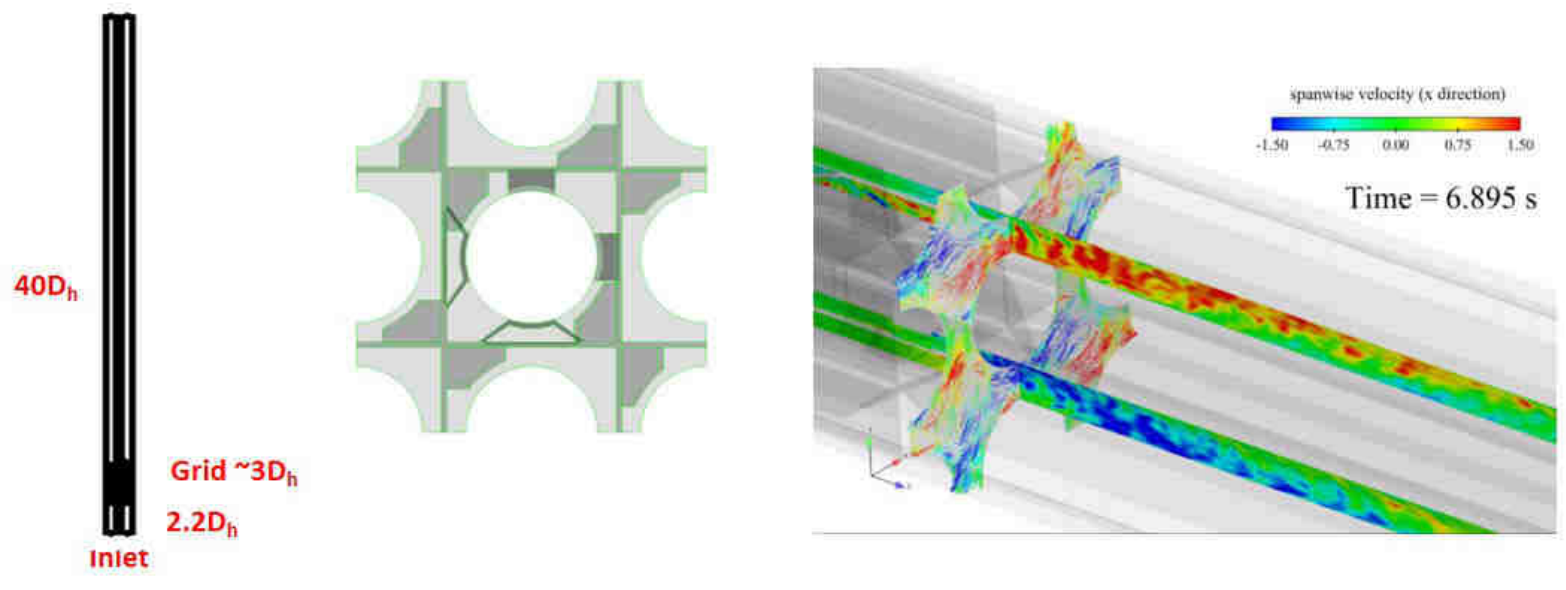

Fig. 10. Flow through a mixing grid at $\mathrm{Re}=40000$. Left: the computational domain, middle: cut of the computational domain $(2 \times 2$ periodic bundle), right: snapshot of the instantaneous crossstream velocity.

\subsection{Towards very large computations?}

Several CFD codes are showing very efficient scalability on very large computers, among them: Code_Saturne open source (code-saturne.org) EDF CFD tool (see Archambeau et al., 2004 and Fournier et al., 2011). This code is based on a collocated finite volume approach. Fig. 11 gives a tentative road-map of the computation sizes that would be used (this is valid for both RANS and LES approaches). One should first distinguish between standard computations (or "production computations") and "Big" computations (could be named "Research computations"). Of course, the objective of these two kinds of simulations is not the same.

Standard computations are today around 10 million cells for a duration of a couple of weeks. Some computations reach the order of 100 million cells in nuclear engineering but are still rare (simulating the whole nuclear vessel for example). The factor that can be gained for this kind of computations in the next five years is not large. A factor of 10 would be very good, considering that standard users will have standard HPC machines with a limited access to the cores and a limited storage memory. It is possible to enhance this projection by enhancing pre and post-processing tools and access to supercomputers. 
"Big computations" will probably continue to grow to reach up to few hundred billion cells or even more with durations of few months. Those who carry out these computations have access to exceptional resources and are rather performing computations using LES or DNS on relatively simple geometries. One should consider also the physical time needed. In the example shown in Fig. 10, the physical time needed to obtain an exploitable pressure load led to run the computation over almost one year on a BlueGene/P with 1024 cores (much more time was needed to predict the pressure load than the statistics). Other physics such as conjugate heat transfer may dramatically increase the physical time needed. In addition, one of the objectives is to increase the Reynolds number. Let's take the example of DNS in a homogeneous isotropic turbulence. If the time step is of the order of the Kolmogorov time scale, increasing the turbulent Reynolds number by a factor of 100 will reduced the time step by a factor of 10 which will make the computation naturally longer. This effect should be less dramatic in LES but there will be also an increase of the needed computational time.

\begin{tabular}{|c|c|c|c|c|}
\hline 2000 & 2005 & 20010 & today & 2020 \\
\hline $\begin{array}{l}1 \text { Million cells } \\
\text { Few months }\end{array}$ & $\begin{array}{l}10 \text { Million cells } \\
\text { Few months }\end{array}$ & $\begin{array}{l}100 \mathrm{M} \text { cellules } \\
\text { Few months }\end{array}$ & $\begin{array}{l}1 \text { billion cells } \\
\text { Few months }\end{array}$ & $\begin{array}{l}10 \text { a } 100 \text { billion cells } \\
\text { Few months }\end{array}$ \\
\hline \multicolumn{5}{|c|}{ «Big » computations } \\
\hline $\begin{array}{l}1 \mathrm{M} \text { cells } \\
2 \text { to } 6 \text { months }\end{array}$ & $\begin{array}{l}1 \mathrm{M} \text { cells } \\
1 \mathrm{month}\end{array}$ & $\begin{array}{l}5 \mathrm{M} \text { cells } \\
1 \mathrm{month}\end{array}$ & $\begin{array}{l}10 \text { M cells } \\
2 \text { weeks }\end{array}$ & $\begin{array}{l}100 \mathrm{M} \text { cells } \\
2 \text { weeks }\end{array}$ \\
\hline
\end{tabular}

Fig. 11. Tentative roadmap of HPC computations using Code_Saturne.

\section{Conclusions and perspectives}

The present paper gives some ideas and guidance one should keep in mind while running (U)RANS or LES computations. Here are the major ones concerning both approaches:

- LEVMs can produce satisfactory results in particular while dealing with global quantities,

- If the flow exhibits anisotropic turbulence due to rotating or secondary flows, one should rather use a RSM, in particular to obtain local quantities,

- Wall-resolved RANS simulations are clearly superior to wall-modeled ones,

- Predicted unsteadiness from (U)RANS simulations should be treated with caution as they might be highly dependent on numerical parameters.,

- Wall refinement is not the only important issue for a wall-resolved LES, the cell sizes far from the wall should be small enough to be in the inertial zone and some a posteriori tests can be performed to check the quality of the computation,

Some perspective can be also drawn:

- RSMs and advanced models for turbulent heat fluxes still need much more development, feedback and dissemination,

- Adaptive wall treatment for RANS in order to deal with very large Reynolds numbers can replace more standard approaches, 
- More DNS and very fine LES should be carried out in the future, in particular on complex geometries, to contribute to fluid flow understanding and to (U)RANS validation,

- Wall modeling in LES will remain questionable,

- The physical time needed for DNS and LES will increase in the future leading to longer computations,

- More efforts (pre-post processing, access to bigger resources) need to be made to increase the size of "standard" computations.

\section{Acknowledgements}

The author would like to acknowledge all the contributors to the numerical simulations shown here. He also warmly acknowledges NURETH-16 organizers who invited the author to give an invited lecture summarized in the present article.

\section{References}

Abe, A., Kawamura, H., Matsuo, Y., 2004. Surface heat-flux fluctuations in a turbulent channel flow up to $\operatorname{Re}_{\tau}=1020$ with $\operatorname{Pr}=0.025$ and 0.71. Int. J. Heat Fluid Flow 25, 404-419.

Afgan, I., Kahil, Y., Benhamadouche, S., Sagaut, P., 2011. Large eddy simulation of the flow around single and two side-by-side cylinders at subcritical Reynolds numbers. Physics of Fluids 23(7), 075101.

Archambeau, F., Mechitoua, N., Sakiz, M., 2004. Code_Saturne: A finite volume method for the computation of turbulent incompressible flows: Industrial applications. International Journal on Finite Volumes, 1(1).

Baglietto, E., Ninokata, H., Misawa, T., 2006. CFD and DNS methodologies development for fuel bundle simulations. Nuclear Engineering and Design 236, Issues 14-16, 1503-1510.

Benhamadouche, S., Moussou, P., Le-Maître, C., 2009. CFD estimation of the flow-induced vibrations of a fuel rod downstream a mixing grid. Proceedings of PVP 2009 ASME Pressure Vessels and Piping 2009 / Creep 8 Conference, July 22-26, Prague, Czech Republic.

Bellet, S., Benhamadouche, S., 2010. Swirling and secondary flows in PWR primary loops, CFD might bring some light. Proceedings of the 18th International Conference on Nuclear Engineering (ICONE18), May 17-21, Xi'an, China.

Benhamadouche, S., 2015. Pressure drop predictions using Code_Saturne in NESTOR CFD Benchmark. Proceedings of the 16th International Topical Meeting on Nuclear Reactor Thermal Hydraulics (NURETH-16), Chicago, Illinois, USA, August 30 - September 4.

Benhamadouche, S., Arenas, M., Malouf, W.J., 2015a. Wall resolved Large Eddy Simulation of a flow through a square-edged orifice in a round pipe at $R e=25000$. Proceedings of the $16^{\text {th }}$ International Topical Meeting on Nuclear Reactor Thermal Hydraulics (NURETH-16), Chicago, Illinois, USA, August 30 - September 4 (submitted in the current Special Issue of Nuclear Engineering and Design).

Benhamadouche, S., Malouf, W.J., Arenas, M., 2015b. Effects of spatial discretisation and RANS turbulence modeling on the numerical simulation of a flow through a square-edged 
orifice in a round pipe. E-proceedings of the 36th IAHR World Congress, 28 June - 3 July, 2015, The Hague, the Netherlands.

Billard, F., Laurence, D., 2012. A robust k- $\varepsilon-\mathrm{v}^{2} / \mathrm{k}$ elliptic blending turbulence model applied to near-wall separated and buoyant flows. Int. J. of Heat and Fluid Flow 33 45-58.

Billard, F., Laurence, D., Osman, K., 2015. Adaptive Wall Functions for an Elliptic Blending Eddy Viscosity Model Applicable to Any Mesh Topology. Flow, Turbulence and Combustion, 94, issue 4, 817-842.

Chabard, J.P., Laurence, D., 2009. Heat and fluid flow simulations for deciding tomorrow's energy, Proceedings of Turbulent, Heat and Mass Transfer 6, 14-18 September, Rome, Italy.

Chang, D., Tavoularis, S., 2008. Simulations of turbulence, heat transfer and mixing across narrow gaps between rod-bundle subchannels. Nuclear Engineering and Design 238, 109-123.

Conner, M.E., Karoutas, Z.E., and Xu Y., 2015. Westinghouse CFD modeling and results for EPRI NESTOR CFD round Robin exercise of PWR rod bundle testing. Proceedings of the $16^{\text {th }}$ International Topical Meeting on Nuclear Reactor Thermal Hydraulics (NURETH-16), Chicago, Illinois, USA, August 30 - September 4.

Craft, T.J., Gant, S.E., Iacovides, H., Launder, B.E., 2004. A new wall function strategy for complex turbulent flows. Numer. Heat Transfer, B, 45, 301-317.

Craft, T.J., Gerasimov, A.V., Iacovides, H., Launder, B.E., 2002. Progress in the generalization of wall functions treatment. Int. J. Heat Fluid Flow 23, 148-160.

Dairay, T., Fortuné, V., Lamballais, E., Brizzi, L.-E.,2015. Direct numerical simulation of a turbulent jet impinging on a heated wall. Journal of Fluid Mechanics, 764, 362-394.

Dehoux, F., 2012. Modélisation statistique des écoulements turbulents en convection forcée, mixte et naturelle. Phd Thesis, Université de Poitiers.

Dehoux, F., Lecocq, Y., Benhamadouche, S., Manceau, R., Brizzi, L.-E., 2011. Algebraic modelling of the turbulent heat fluxes using the elliptic blending approach - application to forced and mixed convection regimes," Flow, Turbulence and Combustion 88 (1-2), 77-100.

Dol, H.S., Hanjalić, K., Versteegh, T.A.M., 1999. A DNS-based thermal second moment closure for buoyant convection at vertical walls. J. Fluid. Mech. 391, 211-247.

J.J. Derksen, J.J., 2005. Simulations of confined turbulent vortex flow. Computers \& Fluids 34 301-318.

Durbin, P.A., 1991. Near-wall turbulence closure modeling without damping functions. Theoret. Comput. Fluid Dynamics 3, 1-13.

Durbin, P.A., 1993. A Reynolds stress model for near-wall turbulence. J. Fluid. Mech. 249, 465-498. 
Esch, T., Menter, F.R., 2003. Heat transfer predictions based on two-equation turbulence models with advanced wall treatment. In Proc. 4th Int. Symp. Turbulence, Heat and Mass Transfer, Antalya, Turkey, 2003.

Escudier, M.P., Bornstein, J., Zehnder, N., 1980. Observations and LDA measurements of confined turbulent vortex flow. Journal of Fluid Mechanics 98, 49-63.

Fadai-Ghotbi, A., Manceau, R., Borée, J., 2008. Revisiting URANS computations of the backwardfacing step flow using second moment closures. Influence of the numerics. Flow, Turbulence and Combustion 81(3), 395-414.

Flageul, S., Benhamadouche, S., Lamballais, E., Laurence, D., 2015. DNS of turbulent channel flow with conjugate heat transfer: Effect of thermal boundary conditions on the second moments and budgets. Int. J. Heat and Fluid Flow 55, 34-44.

Fournier, Y., Bonelle, J., Moulinec, C., Shang, Z., Sunderland, A.G., Uribe, J.C., 2011. Optimizing Code_Saturne computations on Petascale systems. Computers \& Fluids 3(7), 17601765.

Hanjalić, K., Launder, B.E., 2011. Modelling turbulence in engineering and the environment: second moment routes to closure. Cambridge university press.

ISO 5167-2, 2003. Measurement of fluid flow by means of pressure differential devices inserted in circular cross-section conduits running full -- Part 2.

Kasagi, N., Nishimura, M., 1997. Direct numerical simulation of combined forced and natural turbulent convection in a vertical plane channel. Int. J. Heat Fluid Fl., 18(1), 88-99.

Kenjereš, S., Gunarjo, S.B., Hanjalić, K., 2005. Contribution to elliptic relaxation modelling of turbulent natural and mixed convection. Int. J. Heat and Fluid Flow 26, 569-586.

Keshmiri, A., Cotton, M., Addad Y., Laurence, D., 2012. Turbulence Models and Large Eddy Simulations Applied to Ascending Mixed Convection Flows. Flow, Turbulence and Combustion 48(0), 1257-1271.

Keshmiri, A., Osman, K., Benhamadouche, S., Shokri, N., 2016. Assessment of Advanced RANS Models against Large Eddy Simulation and Experimental Data in the Investigation of Ribbed Passages with Passive Heat Transfer. Numerical Heat Transfer, Part B: Fundamentals, In Press.

Kim, W.S., He, S., Jackson, J.D., 2008. Assessment by comparison with DNS data of turbulence models used in simulations of mixed convection. Int. J. of Heat and Mass Transfer 51, 12931312 .

Komen, E.M.J., Shams, A., Camilo, L., Koren, B., 2014. Quasi-DNS capabilities of OpenFOAM for different mesh types. Computers and Fluids, 96 87-104.

Launder, BE., Reece, G.J., Rodi, W., 1975. Progress in the Development of a Reynolds-Stress Turbulence Closure. Journal of Fluid Mechanics 68(3), 537-566. 
Launder, B.E., Sharma, B.I., 1974. Application of the energy dissipation model of turbulence to the calculation of flow near a spinning disc. Lett. Heat Mass Transfer 1, 131-138.

Launder, B.E., Spalding, D.B., 1974. The numerical computation of turbulent flows. Comp. Meth. in Appl. Mech. Eng. 3, 269-289.

Laurence, D., Uribe, J., Utyuzhnikov, S., 2004. A robust formulation of the v2-f model. Flow, Turbulence and Combustion 73(3), 169-185.

Manceau, R., Hanjalić, K., 2002. Elliptic blending model: A new near-wall Reynolds-stress turbulence closure. Phys. Fluids 14(2), 744-754.

Menter, F.R., 1994. Two-Equation Eddy-Viscosity Turbulence Models for Engineering Applications. AIAA Journal, 32(8), 1598-1605.

Meyer, L., 2010. From discovery to recognition of periodic large scale vortices in rod bundles as source of natural mixing between subchannels-a review. Nucl. Eng. Des. 240, 1575-1588.

Piomelli, U., 2008. Wall-Layer Models for Large-Eddy Simulations. Progress in Aerospace Sciences, Large Eddy Simulation - Current Capabilities and Areas of Needed Research, 44, no. $6: 437-46$.

S. B. Pope, S.B., 2000. Turbulent flows. Cambridge university press.

Popovac, M., Hanjalić, K., 2007. Compound wall treatment for RANS computation of complex turbulent flows and heat transfer. Flow Turb. Comb. 78, 177-202.

Ranjan, R., Pantano C., Fischer, P., 2011. Direct simulation of turbulent heat transfer in swept flow over a wire in a channel. International Journal of Heat and Mass Transfer 54 4636-4654.

Shams, A., Roelofs, F., Komen, E.M.J., Baglietto, E., 2013. Quasi-direct numerical simulation of a pebble bed configuration, Part-II: Temperature field analysis. Nucl. Eng. Design 263, 490499.

Shih, T.-H, Liou, W.W., Shabbir, A., Yang, Z., Zhu, J., 1995. A New k- $\varepsilon$ Eddy-Viscosity Model for High Reynolds Number Turbulent Flows - Model Development and Validation. Computers \& Fluids 24(3), 227-238.

Speziale, C.G., Sarkar, S., Gatski, T.B., 1991. Modelling the pressure-strain correlation of turbulence: an invariant dynamical systems approach. Journal of Fluid Mechanics 227, 245272.

Tavoularis, S., 2011. Reprint of: Rod bundle vortex networks, gap vortex streets, and gap instability: A nomenclature and some comments on available methodologies. Nuclear Engineering and Design 241, 4612-4614.

Tiselj, I., Oder, J., Cizelj, L., 2013. Double-sided cooling of heated slab: Conjugate heat transfer DNS. International Journal of Heat and Mass Transfer 66, 781-790. 
Trias, F.X., Soria, M., Oliva, A., Pérez-Segarra, C.D., 2007. Direct numerical simulations of two and three-dimensional turbulent natural convection flows in a differentially heated cavity of aspect ratio 4. J. Fluid Mech. 586, 259-293.

Wald, J.-F., Benhamadouche, S., Manceau, R., 2015. Adaptive wall treatment for the Elliptic Blending Reynolds Stress Model. E-proceedings of the 36th IAHR World Congress, 28 June 3 July, 2015, The Hague, the Netherlands.

Wells, D.M., Peturaud, P., Yagnik, S.K., 2016. Overview of CFD Round Robin Benchmark of the High Fidelity Fuel Rod Bundle NESTOR Experimental Data. Proceedings of the $16^{\text {th }}$ International Topical Meeting on Nuclear Reactor Thermal Hydraulics (NURETH-16), Chicago, Illinois, USA, August 30 - September 4, 2015 (submitted in the current Special Issue of Nuclear Engineering and Design). 